\title{
DYNAMICS OF ARTIFICIAL HELICAL MICROSWIMMERS UNDER CONFINEMENT
}

\author{
Hakan Osman Caldag \\ Sabanci University \\ Istanbul, Turkey
}

\author{
Serhat Yesilyurt \\ Sabanci University \\ Istanbul, Turkey
}

\begin{abstract}
Understanding trajectories of natural and artificial helical swimmers under confinement is important in biology and for controlled swimming in potential medical applications. Swimmers follow helical or straight trajectories depending on whether the helical tail is pushing or pulling the swimmer. To investigate swimming dynamics of helical swimmers further, we present a Computational Fluid Dynamics (CFD) model for simulation of an artificial microswimmer in cylindrical channels. The microswimmer has a cylindrical head and a lefthanded helical tail. The kinematic model solves for the position and rotation of the swimmer based on the linear and angular velocities of the force-free swimmer from a CFD model. Thirdorder Adams-Bashforth solver is used to obtain the orientation and the position of the swimmer. Viscous, gravitational, magnetic and contact forces and torques are considered in the model. The model is validated with experimental results. 3D trajectories, propulsion and tangential velocities are reported.
\end{abstract}

\section{INTRODUCTION}

Artificial micro swimmers have huge potential in targeted drug delivery, non-invasive surgery and cell manipulation. Magnetized artificial swimmers with helical tails, inspired by bacteria such as Escheria coli, can be externally actuated using a rotating magnetic field, eliminating the need for on-board apparatus to generate propulsion $(1,2)$. Developments in microfabrication methods have made it possible to produce microswimmers on the order of nanometers (3).

Understanding the trajectories of the swimmers is important in controlled swimming applications. Lauga et al. (4) report that bacteria swim in circular trajectories under force-free and torque-free swimming conditions. For artificial swimmers under confinement, two distinct trajectories stand out, one of which is a helical trajectory close to the channel boundaries when the tail is pushing the head (called pusher-mode) and a straight trajectory close to the center of the channel when the tail pulls the head (called puller-mode) (5). Another factor affecting the trajectories of artificial swimmers is the step-out behavior in which the magnetically actuated swimmer loses its synchronization with the rotating magnetic field due to high viscous torque (5).

Computational analysis tools are helpful in understanding microswimmer motion. Zhu et al. (6) obtain trajectories for lowReynolds number swimming of squirmers in circular channels using boundary element method (BEM). Regularity of the motion of spherical swimmers in a cylindrical Poiseuille flow are shown to be dependent on small finite periodic oscillations that vary with the position and orientation of the swimmer in the channel and also efficient upstream (downstream) swimming takes place at (away from) the center. Zöttl and Stark (7) use a dipole approximation to study the stability of a pointlike swimmer. They find a pusher tends to follow a circular trajectory around the centerline of the channel, close to channel boundaries. Pullers, on the other hand, follow a straight path at the center of the channel (7). Another study by the same authors reveal that changing the aspect ratio of a spheroidal swimmer only changes the frequency of oscillatory trajectory (8). Studies on helical swimmers explain wobbling by the imbalance of drag forces on the tail and head which create a torque that pushes the swimmer to follow a circular path (4). Wobbling is related with low Mason number (Ma) which is the ratio of hydrodynamic torque to magnetic torque (9).

In our previous work, we used steady Stokes equations and demonstrated the effects of geometric parameters on forward and lateral instantaneous velocities and also wobbling rates of microswimmers composed of a magnetic head and a helical tail (10). The ideal geometry of singly flagellated bacteria and critical channel radius were investigated for the maximum 
swimming velocity and efficiency (10). Experimental trajectories of mm-sized artificial helical swimmers inside circular channels are reported with respect to amount of confinement, tail length, magnetic field rotation rate and fluid flowrate (5).

This paper presents a kinematic model that uses computational fluid dynamics (CFD) for the simulation of a helical microswimmer inside cylindrical channels. Swimmer position and orientation are evaluated from integration of linear and angular velocities which are calculated from CFD using force-free and torque-free swimming conditions. Viscous, magnetic, gravitational and contact forces are represented in the model. The model is validated with previous experimental results, discussing swimmer velocity and trajectories in pusher and puller modes. Results confirm our experimental observations. The model will be used to investigate swimming dynamics under confinement.

\section{NOMENCLATURE}

$D_{h}$ Diameter of the cylindrical head

$B$ Amplitude of the helical wave

$L_{h} \quad$ Length of the head

$D_{t} \quad$ Diameter of the tail

$L \quad$ Length of the tail

$L_{o} \quad$ Total length of the swimmer

$D_{c h}$ Channel diameter

x Position vector of a point on the swimmer

u Velocity vector

$\mathbf{e}_{i}$ Local coordinate system unit vectors for $\mathrm{i}=1,2,3$

$\omega$ Angular velocity vector

$\mathbf{F}_{v}$ Viscous force on the swimmer

$\mathbf{F}_{w}$ Swimmer weight

$\mathbf{F}_{c}$ Contact force

$\boldsymbol{\tau}_{v} \quad$ Viscous torque on the swimmer

$\boldsymbol{\tau}_{m}$ Magnetic torque on the swimmer

$\boldsymbol{\tau}_{w}$ Torque on the swimmer due to its weight

$\boldsymbol{\tau}_{c}$ Torque on the swimmer due to contact with channel

M Magnetization vector $\left[\mathrm{A} \cdot \mathrm{m}^{2}\right]$

B Magnetic field vector [T]

$\mathrm{B}_{0} \quad$ Magnitude of rotating magnetic field [T]

$\omega$ Rotation rate of magnetic field [ $\mathrm{rad} / \mathrm{s}]$

$t \quad$ Time [s]

m Magnetic moment $[\mathrm{A} / \mathrm{m}]$

$V \quad$ Magnet volume $\left[\mathrm{m}^{3}\right]$

$\mathbf{B}_{\mathbf{R}}$ Remanence vector of the magnet [T]

$\mu_{0}$ Permeability of vacuum $[\mathrm{H} / \mathrm{m}]$

$\sigma_{i y}$ Cartesian components of stress in $\mathbf{y}$ - direction

$\sigma_{i z}$ Cartesian components of stress in $\mathbf{z}$ - direction $\mathbf{n}_{i}$ Components of surface normal vectors

$\theta$ Angular position of a point on swimmer in cylindrical coordinates

$\hat{\mathbf{e}}_{\mathbf{r}} \quad$ Unit vector in $\mathbf{r}$ - direction

$S$ Swimmer surface

$F_{r} \quad$ Total radial force acting on the swimmer

$r_{t} \quad$ Radius of tail filament

$R_{c h}$ Channel radius

$r \quad$ Radial position of a point on the swimmer

$\delta_{w}$ Threshold value of distance between a point on swimmer and channel wall which indicates there is contact

$\sigma_{\text {wall }}$ Contact stress on swimmer

Re Reynolds number

$p$ Pressure

$\rho \quad$ Fluid density $\left[\mathrm{kg} / \mathrm{m}^{3}\right]$

$L_{\text {scale }}$ Length scale for nondimensionalization [m]

$f \quad$ Rotation frequency of the magnetic field [Hz]

$\mu \quad$ Fluid viscosity $[\mathrm{Pa} \cdot \mathrm{s}]$

$t_{\text {scale }}$ Time scale $[\mathrm{s}]$

V Swimmer velocity vector (without rotation)

$U$ Swimmer velocity in $\mathbf{x}$ - direction (without rotation)

$V \quad$ Swimmer velocity in $\mathbf{y}$-direction (without rotation)

$W$ Swimmer velocity in $\mathbf{z}$ - direction (without rotation)

$\omega_{x}$ Angular velocity in $\mathbf{x}$ - direction

$\omega_{y}$ Angular velocity in $\mathbf{y}$ - direction

$\omega_{z}$ Angular velocity in z- direction

$\mathbf{x}_{c} \quad$ Center-of-mass coordinates of the swimmer

$\beta$ Non-dimensional radial position

$R_{h} \quad$ Swimmer head radius

$u_{s w}$ Non-dimensional $\mathbf{x}$ - direction velocity of the swimmer

$v_{\theta, s w}$ Non-dimensional lateral velocity of the swimmer

$v_{s w}$ Non-dimensional swimmer velocity in $\mathbf{y}$-direction

$w_{s w}$ Non-dimensional swimmer velocity in $\mathbf{z}$ - direction

\section{METHODOLOGY}

The geometric model is shown in Fig. 1 where a swimmer with a helical tail and cylindrical head is placed inside a circular channel of diameter $D_{c h}$. Swimmer tail length is $L$, overall length of the swimmer is $L_{o}$, the wavelength and amplitude of the tail are $\lambda$ and $B$, and the diameter of the filament tail is $D_{t}$. The cylindrical head has a length of $L_{h}$ and a diameter of $D_{h}$. The values for these parameters are the same as the values of swimmers used in experiments in our previous work and listed in Table 1 (11,5). Pusher and puller-mode swimming are depicted in the negative and positive $\mathbf{x}$ - directions respectively in Fig. 1. 


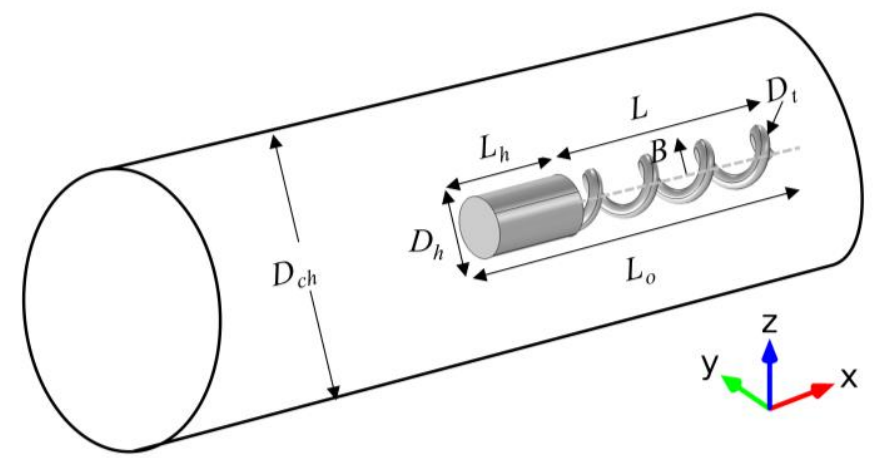

Figure 1: Forward (head direction) and backward (tail direction) motion of the swimmer and geometric parameters of the swimmer model.

Table 1. Geometric parameters of the swimmer.

\begin{tabular}{|c|c|}
\hline Parameter & Values \\
\hline Diameter of the cylindrical head, $D_{h}$ & $0.8 \mathrm{~mm}$ \\
\hline Amplitude of the helical wave, $B$ & $0.4 \mathrm{~mm}$ \\
\hline Length of the head, $L_{h}$ & $1.5 \mathrm{~mm}$ \\
\hline Diameter of the tail, $D_{t}$ & $0.2 \mathrm{~mm}$ \\
\hline Length of the tail, $L$ & $4 \mathrm{~mm}$ \\
\hline Total length of the swimmer, $L_{o}$ & $5.5 \mathrm{~mm}$ \\
\hline Channel diameter, $D_{c h}$ & $1.6 \mathrm{~mm}$ \\
\hline
\end{tabular}

Swimmer motion can be expressed by two basic equations of motion:

$$
\begin{aligned}
& \frac{d \mathbf{x}}{d t}=\mathbf{u} \\
& \frac{d \mathbf{e}_{i}}{d t}=\boldsymbol{\omega} \times \mathbf{e}_{i}
\end{aligned}
$$

where $\mathbf{u}$ and $\boldsymbol{\omega}$ are linear and angular velocity vectors of the swimmer, $\mathbf{e}_{i}$ for $i=1,2,3$ denotes the local coordinate system placed at the center of mass of the swimmer. Coordinate frames are shown at Fig. 2. Low Reynolds number swimming means inertial effects are negligibly small, and forces and torques acting on the swimmer can be taken as zero. This condition, known as force-free and torque-free swimming, is used to evaluate $\mathbf{u}$ and $\boldsymbol{\omega}$ :

$$
\begin{aligned}
& \mathbf{F}_{v}+\mathbf{F}_{w}+\mathbf{F}_{c}=0 \\
& \boldsymbol{\tau}_{v}+\boldsymbol{\tau}_{m}+\boldsymbol{\tau}_{w}+\boldsymbol{\tau}_{c}=0
\end{aligned}
$$

where subscript $v$ stands for viscous, $m$ for magnetic, $w$ for gravity and $c$ for contact. Viscous force on the swimmer can be obtained by the integration of fluid stress on the swimmer. A magnetic field rotating on the $y-z$ plane is applied to rotate the swimmer in the experiments to generate the torque that will rotate the swimmer around $\mathbf{x}$ - axis. As the magnetic field rotates, a magnetic torque applies to the swimmer:

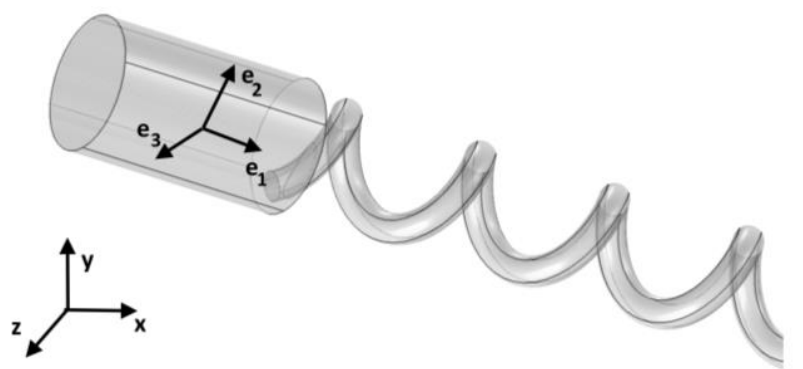

Figure 2: Placement of local and global coordinate system.

$$
\boldsymbol{\tau}_{\mathbf{m}}=\mathbf{M} \times \mathbf{B}
$$

where $\mathbf{B}=\left[\begin{array}{ll}0 & B_{0} \cos (\omega t) B_{0} \sin (\omega t)\end{array}\right]$ ' and $\mathbf{M}$ is the magnetization vector of the swimmer in $A \cdot \mathrm{m}^{2}$. Sign of $\omega$, rotation rate, implies the rotation direction of the swimmer (positive for pusher-mode and negative for puller-mode), $t$ is time. Magnetization is calculated from $\mathbf{M}=\mathbf{m} V$ where $\mathbf{m}$ is the magnetic moment $(\mathrm{A} / \mathrm{m})$ and $V$ is the magnet volume. Magnetic moment is calculated from $\mathbf{m}=\mathbf{B}_{\mathbf{R}} / \mu_{0}$ where $\mathbf{B}_{\mathbf{R}}$ is the remanence of the magnet (with a magnitude of around $1 \mathrm{~T}$ for $\mathrm{NdFeB}$ magnets) and $\mu_{0}$ is permeability of free space in $\mathrm{H} / \mathrm{m}$ (12).

Head and tail weights are calculated separately because neodymium magnet is denser than the plastic polymer making up the tail. Buoyancy force is subtracted from the weights. Weights are taken as point forces acting at the center-of-mass of the head and tail in torque calculations.

Swimmer is assumed to be in contact with the channel wall when the distance between the surface of the swimmer body to the channel wall is less than $r_{t}$, radius of tail filament. In the simulation results to be discussed, tail radius is $10 \%$ of the channel radius, so the swimmer motion is not restricted significantly. Contact force is applied in the normal direction to the channel wall at an amount of the radial force applied by the swimmer onto the fluid. Radial stress $\boldsymbol{\sigma}_{r}$ is obtained from the stresses in $\mathbf{y}$ - and $\mathbf{z}$ - directions:

$$
\boldsymbol{\sigma}_{r}=\left(\sigma_{i y} \mathbf{n}_{i} \cos (\theta)+\sigma_{i z} \mathbf{n}_{i} \sin (\theta)\right) \hat{\mathbf{e}}_{\mathbf{r}}
$$

where $\sigma_{i y}$ and $\sigma_{i z}$ for $i=x, y, z$ are the stress components in $\mathbf{y}$ - and z- directions, respectively, $\theta=\operatorname{atan} 2(z, y)$ where $z$ and $y$ denote $\mathbf{y}$ - and $\mathbf{z}$ - coordinates of a point on swimmer surface, $\mathbf{n}_{i}$ for $i=1,2,3$ are surface normals and $\hat{\mathbf{e}}_{\mathbf{r}}$ is the unit vector in radial direction in the channel and summation over the repeated indices is implied. Integration of stress over swimmer surface $S$ gives net radial force:

$$
F_{r}=-\int_{S} \boldsymbol{\sigma}_{r} \cdot \mathbf{n} d S
$$

where $\mathbf{n}$ is the surface normal. The integration is multiplied with a minus sign to find the force on the swimmer. Contact force is 
applied on the parts where the radial position is beyond the threshold and if the radial force is towards the channel wall. These two conditions are satisfied with the following Boolean checks in contact force per unit area formulation:

$$
\sigma_{\text {wall },\{y, z\}}=\sigma_{\{y, z\}}\left(F_{r}>0\right)\left(R_{c h}-r<\delta_{w}\right)
$$

where $R_{c h}$ is channel radius and $r$ is the radial position of a point on the swimmer in the $\mathrm{y}-\mathrm{z}$ plane. $\delta_{w}$ is the threshold value that constitutes the distance of the contact region from the channel wall, which is equal to $r_{t}$. Wall contact force will be applied if $F_{r}$ is towards channel wall, i.e., the swimmer is moving towards the wall, meaning $F_{r}$ should be positive.

Fluid motion around the swimmer is governed by incompressible Stokes equations as the Reynolds number is much less than unity:

$$
\frac{1}{R e} \nabla^{2} \mathbf{u}-\nabla p=0, \quad \nabla \cdot \mathbf{u}=0
$$

Here, $\mathbf{u}$ and $p$ are the nondimensional velocity vector and the pressure, respectively, and $\mathrm{Re}$ is the Reynolds number defined as $\operatorname{Re}=\rho L_{\text {scale }}^{2}|f| / \mu$ where $L_{\text {scale }}$ is an appropriate length scale of the non-dimensional model, $1 \mathrm{~mm},|f|=|2 \pi \omega|$ is the rotation frequency of the magnetic field, $\rho$ and $\mu$ are density and viscosity of the fluid, respectively. Its reciprocal, $1 /|f|$, is the time scale of the model, $t_{\text {scale }}, \mu$ is fluid viscosity, pressure is nondimensionalized with the group $\rho L_{\text {scale }}^{2} / t_{\text {scale }}^{2}$ and $\mathbf{u}$ is nondimensionalized with $L_{\text {scale }} / t_{\text {scale }}$. The forces are nondimensionalized with the group $\rho L_{\text {scale }}^{4} / t_{\text {scale }}^{2}$ and torques are nondimensionalized with the group $\rho L_{\text {scale }}^{5} / t_{\text {scale }}^{2}$.

Channel wall and swimmer surface have no-slip boundary conditions. Swimmer surface moves with a velocity of $\mathbf{V}=[U$, $V, W]^{\prime}$ and rotates with the angular velocity vector, $\boldsymbol{\omega}=\left[\omega_{x}, \omega_{y}\right.$, $\left.\omega_{z}\right]^{\prime}$. Velocity of a point on swimmer surface is specified as:

$$
\mathbf{u}=\mathbf{V}+\boldsymbol{\omega} \times\left(\mathbf{x}-\mathbf{x}_{c}\right)
$$

where $\mathbf{x}_{c}$ is the position of center of mass. Both ends of the cylinder are closed. Commercial finite-element software COMSOL is used to solve the Stokes equations numerically to obtain for $\mathbf{u}$ and $\boldsymbol{\omega}$ under the torques and forces that act on the swimmer (13). Fluid domain is discretized with tetrahedral elements and triangular elements are used for the swimmer surface in meshing. Meshing on swimmer body is denser than the meshing on the fluid. Overall mesh consists of 75090 elements with 69784 degrees of freedom for a swimmer with a tail length of $4 \mathrm{~mm}$. A convergence test is carried out with results shown in Table 2. Error in $v_{s w}$ and $w_{s w}, \mathbf{y}-$ and zdirection velocities, are high on coarse meshing but the values are close to the densest mesh results in the chosen mesh density while solving ten times faster.
Table 2. Convergence test for the CFD model. The line in bold is the meshing density used for simulations.

\begin{tabular}{|c|c|c|c|c|c|}
\hline $\begin{array}{c}\text { Number } \\
\text { of domain } \\
\text { elements }\end{array}$ & $\begin{array}{c}\text { Degrees } \\
\text { of } \\
\text { freedom }\end{array}$ & $\begin{array}{c}\text { Computation } \\
\text { Time }[\mathrm{s}]\end{array}$ & $\begin{array}{c}\text { Error in } \\
u_{s w}[\%]\end{array}$ & $\begin{array}{c}\text { Error in } \\
v_{s w}[\%]\end{array}$ & $\begin{array}{c}\text { Error in } \\
w_{s w}[\%]\end{array}$ \\
\hline 45830 & 44996 & 8 & -4.14 & -5.2 & -13.7 \\
\hline $\mathbf{7 5 0 9 0}$ & $\mathbf{6 9 7 8 4}$ & $\mathbf{1 1}$ & $\mathbf{- 0 . 0 7}$ & $\mathbf{0 . 6}$ & $\mathbf{- 2}$ \\
\hline 172749 & 147584 & 21 & -0.02 & 0.1 & -0.3 \\
\hline 306077 & 247213 & 43 & -0.02 & 0.2 & -0.1 \\
\hline 520218 & 404733 & 97 & $-*$ & $-*$ & $-*$ \\
\hline *Error is defined with respect to the results from simulations \\
with the densest meshing. \\
\hline \multicolumn{7}{|c|}{} \\
\hline \multicolumn{7}{|c|}{} \\
\hline
\end{tabular}

Linear and angular velocities are obtained from the CFD simulation for given position and orientation of the swimmer and used to update position and orientation at the next time step according to (1) and (2) with the Adams-Bashforth integration.

\section{RESULTS}

Swimmer trajectories and velocities are compared with experimental results from our previous work (5). Initial position of the swimmer is set closer to the bottom of the channel to mimic the swimmer that rests on the wall due to its weight. The swimmer orientation is assumed to be along the channel's long axis. Rotation frequency of the magnetic field is set to $15 \mathrm{~Hz}$ where we observe synchronous rotation of the swimmer with the magnetic field and differences in pusher and puller-mode swimming. Figs. $3 \mathrm{a}$ and $3 \mathrm{~b}$ shows $3 \mathrm{D}$ trajectory and radial position of the swimmer in pusher-mode simulation (displayed in red). Here, we use non-dimensional radial position, defined as:

$$
\beta=\frac{r_{s w}}{R_{c h}-R_{h}}
$$

where $R_{h}$ is the radius of the head. Non-dimensional $\beta$ is plotted with respect to non-dimensional time, $\omega t / 2 \pi$. The swimmer is following a helical path, just as in the experiments, whose results are shown in blue. The swimmers have a left-handed helical tail and follow a right-handed helical trajectory as the magnetic field rotates in counter-clockwise direction. The trajectory converges to a constant $\beta$ value. Oscillations in $\beta$ value in experiments are because of the swimmer circulating slightly off-center (5).

Puller-mode simulation results also agree with experiments, shown in Figs. 3c and 3d. An important observation from puller-mode simulation is that even though the puller starts its motion off-center, it moves towards the centerline of the channel agreeing with the puller's trajectory from the experiment. The match between the simulations and experiments confirms the distinction between pusher and pullermode swimming. 

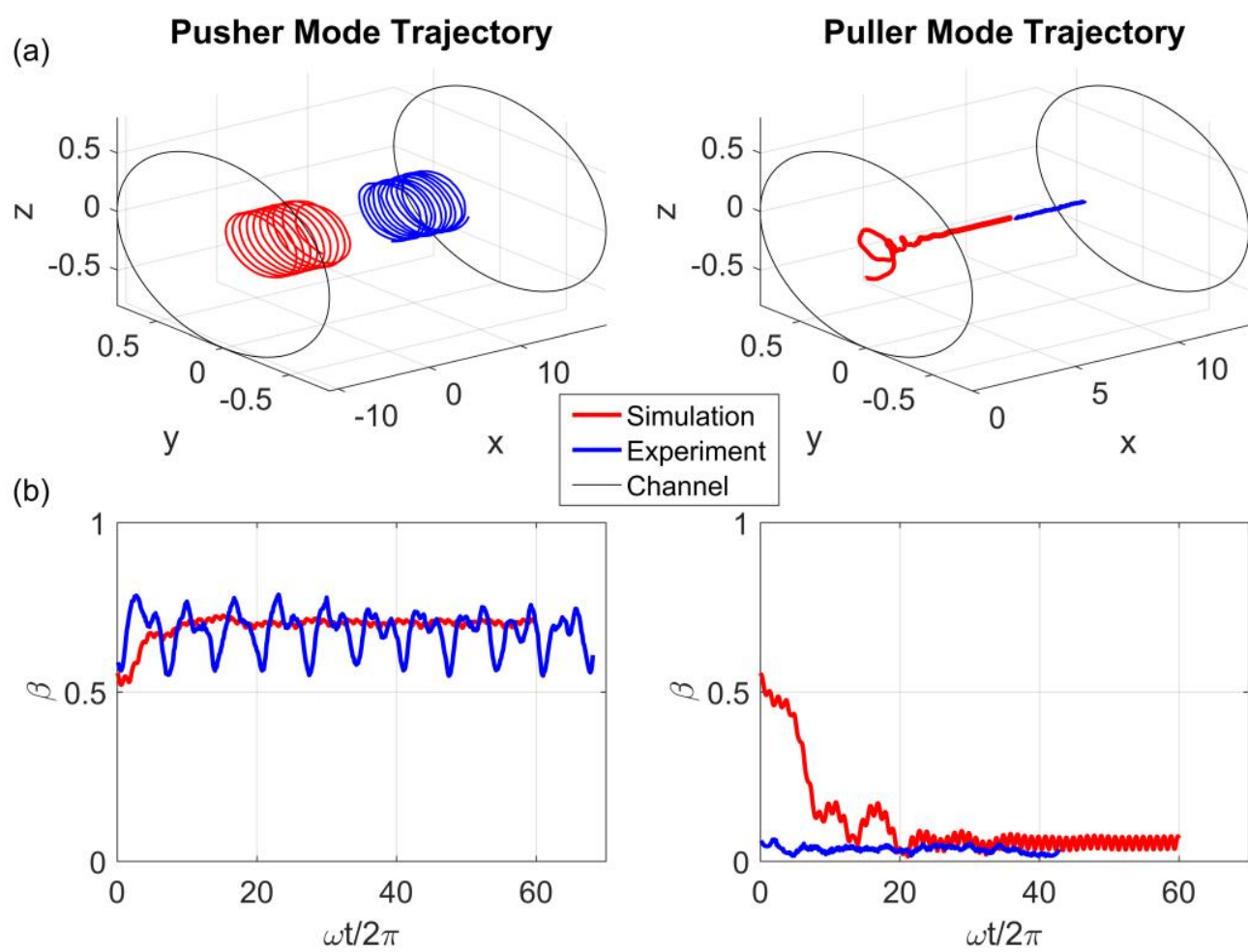

Puller Mode Trajectory

(c)

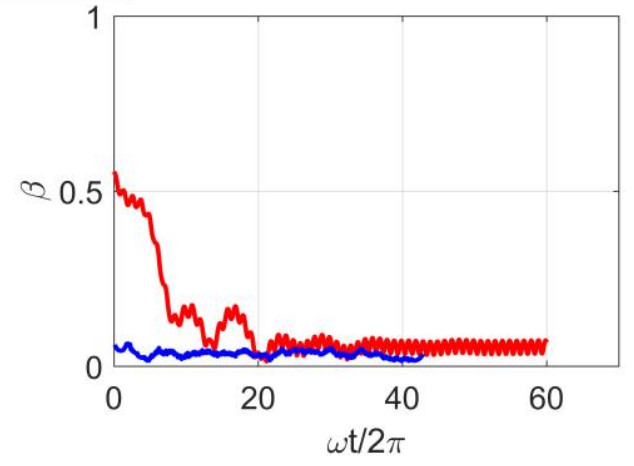

Figure 3: (a) 3D trajectories of pushers in experiment (in blue) and simulation (in red). (b) Change of $\beta$ for pushers in simulation and experiment. (c) 3D trajectories of pullers in experiment and simulation. (d) Change of $\beta$ for pushers in simulation and experiment.

Several aspects of swimmer instability are studied in the literature. Wobbling is observed in biological swimmers, but it is attributed to opposite rotation directions of head and the tail (4). Another study explains wobbling of artificial helical swimmers nearby a surface with the imbalance in local drag forces acting on the helix but there is no discussion on swimming modes and swimming under confinement (14). Man \& Lauga's (9) analytical solution for rotating helices show that wobbling is irrespective of the presence of a head under free swimming conditions. However, the dynamics of the head play an important role in wobbling when the swimmer is under confinement. It was observed in experiments that the tail remains closer to the center of the channel than the swimmer head in the pusher-mode and the simulation results agree with this observation as well, indicating that the suppression of tail wobbling is more pronounced than the viscous shear on the head (5). On the other hand, the puller remains aligned with the long axis of the channel after it moves sufficiently close to the centerline of the channel and it remains close to the center. The distinction is similar to what Zöttl and Stark (7) observe which is explained by swimmer-wall interactions. Vorticity induced by the wall pushes puller towards the center of the channel but in pusher-mode the swimmer is attracted by the wall when it is close to the center and repelled when it is close to the wall, resulting in swinging motion (7).
Fig. 4 shows non-dimensional swimming and lateral velocities for pusher and puller modes. Non-dimensional swimming velocity, $u_{s w}$, is taken as the velocity in $\mathbf{x}$ - direction. $u_{s w}>0$ for downstream puller-mode swimming and $u_{s w}<0$ for upstream pusher-mode swimming. Lateral swimming velocity, $v_{\theta, s w}$, is defined as:

$$
v_{\theta, s w}=-v_{s w} \sin \theta+w_{s w} \cos \theta=\omega_{x} r_{s w}
$$

$v_{\theta, s w}$ is positive in counter-clockwise direction when looked from the head side of the swimmer. $v_{\theta, s}$ tends to 0 in pullermode both in experiment and simulation since the swimmer is nearly following a straight trajectory at the center of the channel (not shown). $u_{s w}$ is overestimated in simulations with a relative error of around $40 \%$ for both cases. Experimental imperfections such as roughness on the tail, shape of the head, collisions with the channel wall may have contributed to slower swimming in experiments. It was observed in previous studies that higher $u_{s w}$ can be achieved when the swimmer is swimming close to the channel wall rather than swimming at the center $(5,10)$. This observation explains the decrease in $u_{s w}$ in puller-mode as the swimmer moves towards the center. Furthermore, the variation in $u_{s w}$ is much less in the puller-mode compared to the pushermode swimming due to better alignment of the swimmer and smaller variation of the radial position of the swimmer. $v_{\theta, s w}$ values from simulations are much closer to experiment values but with higher oscillations. 

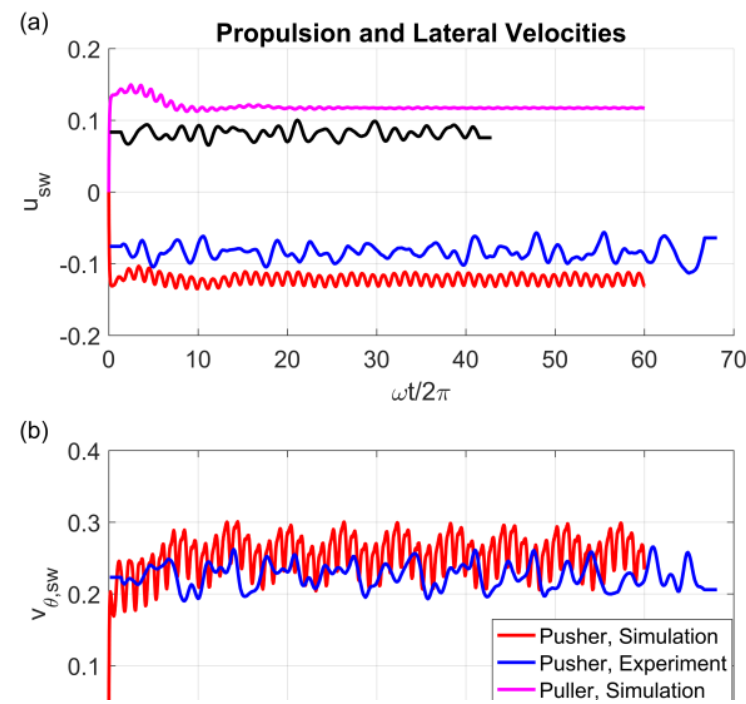

Figure 4: (a) Simulation and experiment results for nondimensional propulsion velocity, $u_{s w}$. (b) Simulation and experiment results for non-dimensional tangential velocity, $v_{\theta, s w}$.

\section{CONCLUSION}

Low Reynolds swimming dynamics of an artificial microswimmer with a helical tail and a cylindrical head inside a cylindrical channel is reported here. A kinematic model is used to obtain the position based on linear and angular velocities from CFD simulations. 3D trajectory of a helical swimmer inside a channel is obtained. Force-free and torque-free swimming conditions are applied in solving Stokes equations. Viscous, gravitational, magnetic and wall contact forces are included in the model. Helical trajectories are observed for the pusher-mode while pullers follow straight trajectories at the center as observed in our previous experiments. Pullers move towards the center of the channel even if they start away from the center. The tail remains closer to the center than the head in the pusher-mode indicating that the tail may be more stable than the head. Comparison of swimming and lateral velocities show the simulation model overpredicts the swimming velocity probably due to imperfections in experiments while tangential velocity is very close. Swimmer is observed to swim slower at the center compared to swimming near wall. It is aimed to use this computational tool to investigate the differences in swimming dynamics to explain the distinction between the modes.

\section{REFERENCES}

(1) Zhang, L., Abbott, J. J., Dong, L., Peyer, K. E., Kratochvil, B. E., Zhang, H., ... \& Nelson, B. J. (2009). Characterizing the swimming properties of artificial bacterial flagella. Nano Letters, 9(10), 3663-3667.
(2) Tottori, S., Zhang, L., Qiu, F., Krawczyk, K. K., Franco-Obregón, A., \& Nelson, B. J. (2012). Magnetic helical micromachines: fabrication, controlled swimming, and cargo transport. Advanced materials, 24(6), 811-816.

(3) Ghosh, A., \& Fischer, P. (2009). Controlled propulsion of artificial magnetic nanostructured propellers. Nano letters, 9(6), 2243-2245.

(4) Lauga, E., DiLuzio, W. R., Whitesides, G. M., \& Stone, H. A. (2006). Swimming in circles: motion of bacteria near solid boundaries. Biophysical journal, 90(2), 400412.

(5) Caldag, H. O., Acemoglu, A. \& Yesilyurt, S. (2017). Experimental characterization of helical swimming trajectories in circular channels. Microfluidics and Nanofluidics 21:136. Springer Berlin Heidelberg https://doi.org/10.1007/s10404-017-1973-9

(6) Zhu, L., Lauga, E., \& Brandt, L. (2013). Low-Reynoldsnumber swimming in a capillary tube. Journal of Fluid Mechanics, 726, 285-311.

(7) Zöttl, A., \& Stark, H. (2012). Nonlinear dynamics of a microswimmer in Poiseuille flow. Physical review letters, 108(21), 218104.

(8) Zöttl, A., \& Stark, H. (2013). Periodic and quasiperiodic motion of an elongated microswimmer in Poiseuille flow, Eur. Phys. J. E., 36 (4).

(9) Man, Y., and Lauga, E. (2013). The wobbling-to-swimming transition of rotated helices. Physics of Fluids, 25(7), 071904

(10) Acemoglu, A., \& Yesilyurt, S. (2014). Effects of Geometric Parameters on Swimming of Micro Organisms with Single Helical Flagellum in Circular Channels. Biophysical Journal, 106(7), 1537-1547.

(11) Acemoglu, A. \& Yesilyurt, S. (2015). Effects of poiseuille flows on swimming of magnetic helical robots in circular channels. Microfluidics and Nanofluidics, 19(5), 1109-1122.

(12) SuperMagnetMan (n.d.) Typical Magnetic Properties of Sintered NdFeB Materials https://cdn.shopify.com/s/files/1/0994/8998/files/Mater ial_tables_on_website_-Neodymium_2.pdf?3243838301807853458

(13) COMSOL AB, Comsol Multiphysics User's Guide, 2017.

(14) Peyer, K. E., Zhang, L., Kratochvil, B. E. \& Nelson, B. J. (2010). Non-ideal swimming of artificial bacterial flagella near a surface. 2010 IEEE International Conference on Robotics and Automation, Anchorage, AK, 2010, pp. 96-101. doi: 10.1109/ROBOT.2010.5509602. 